\title{
The Periodicities of Solar X-ray Flares and Coronal Mass ejections during Solar Cycle 23
}

\author{
Ahmed Abdel Hady \\ Astronomy and Meteorology Department, Faculty of Science, Cairo University, Giza, Egypt, \\ E-mail : aahady@main-scc.cairo.edu.eg
}

\begin{abstract}
The Events of energetic particles from solar X-ray flares and shock waves have been studied. The data were taken from the National Geophysical data center (NGDC) in Boulder, Colorado, USA, Where the data were taken during the solar cycle 23rd. The 23rd cycle is the present one, that started in April 1996, and its maximum was in May 2001, and it will be decayed during year 2007. Power spectrum methods have been applied for analysis of the data given, to find the short and intermediate periodicities. The periodicity around 14 days has appeared in this analysis. This has important implications for understanding and predicting the effects of solar activity on the Earth and on the earth's atmosphere. If a Coronal Mass Ejections (CMEs) hit the Earth, it can excite a geomagnetic storm. Large geomagnetic storms, among other things, can cause electrical power which can damage satellite communications. In space CME typically drive shock waves that produce energetic particles that can damage both electronic equipment and astronauts that are outside the protection of the Earth's magnetic field. So, the prediction of the high energetic particle events is of vital importance for space navigation and airline disasters.
\end{abstract}

\section{Method and Analysis}

The data of events occurred during solar cycle 23rd, were taken from the National Geophysical data center (NGDC) in Boulder, Colorado, USA. The method of power spectrum analysis of the data has been used for all data analysis, where the squared amplitudes $\left[a_{\nu}\right]^{2}$ are taken as the power of the frequency $\nu$, as follows:

$$
\left[a_{\nu}\right]^{2}=\frac{\left(\sum_{i=0}^{N-1} r(i) \cos (2 \pi i)\right)^{2}+\left(\sum_{i=0}^{N-1} r(i) \sin (2 \pi i)\right)^{2}}{N^{2}}
$$

Where $\mathrm{N}$ is the total number of X-ray flares events, equal to 6483 events in solar Xray calculations of power spectrum. By using hamming function the data have been smoothed, where the resolution power spectrum given as:

$$
r_{n}=0.23 r_{n-1}+0.54 r_{n}+0.23 r_{n+1}
$$

For more details about the method of analysis, see for example, Hady A. (2002), and Rieger et al (1984). The relative error values are calculated according to:

$$
R E=\left(2 X-X^{2}\right)^{\frac{1}{2}}
$$

where $R E$ is called the relative error. See for more details, Deeming (1975). The data of X-ray flares events during solar cycles 23 events during 5 years until Dec. 31st, 2002, were analyzed by power spectrum analysis method and given in figure (1), Periodicities of 154.6 days, 52.5 days, 35.4 days, 28 days and 14 days has appeared in the figure. These periodicities are significant because their powers are higher than 0.05 (the level of relative error). These periodicities has appeared and significant from solar X-ray Flare analysis. This implies that the solar X-ray flare is affected by the variation of solar activities and get us a good indication of Ejection of Solar Energetic particles (SEP) in general. 


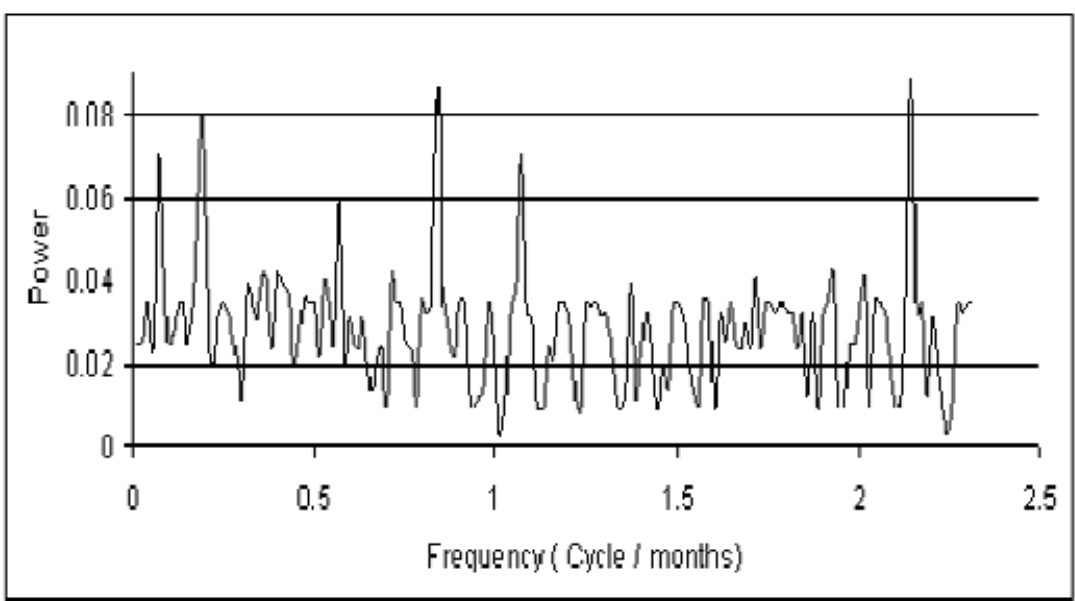

Figure 1. Power spectrum analysis of X-ray flares events during 23rd Solar Cycle until dec. 2002

The data of Coronal Mass Ejections during Solar Cycle 23, have been studied too. Only the periodicity of 154 days has appeared in both types of data, X-ray flare and Coronal Mass Ejections.

\section{Conclusions}

The Power spectrum analysis and the Fast Fourier Power spectrum Transformation, were used for data analysis. The results indicate the periodicities of 1.3 year, 154.6 days, 52.5 days, 35.4 days, 28 days and 14 days. The Periodicity of 154.6 days appears clearly, during the present solar cycle (number 23). The Periodicity of 154.6 days was appeared and reported during the solar cycles 19 and 20 but did not appear during the solar cycles 21 and 22. The absence during solar cycles 21 and 22 is confirmed by a lot of literature, and may be due to the poor activities during these two cycles ,see for example Hady A. (2002). No satisfactory theory exists for the circa 154 days periodicity but several suggestions have been made. This periodicity results from strong magnetized streams appearing in stack plots of synoptic magnetic charts has been refuted by Bai and Sturrock (1991). The other periodicities are the reflects of the effect of solar activity on the rate of Solar energetic particles ejections. The majority of short-term periodicities are of solar origin, perhaps resulting from the deep rotation of the solar core; and the other layers of the Sun.

\section{References}

Bai, T. and Sturrok P. A. 1991 Nature 350, 141.

Abdel Hady A. 2002 Planetary and Space Science J. 50, 89-92.

Deeming T. J. 1975 Astrophysics and Space Science 36, 137.

Rieger, E.,Share, G.,H., Forrest D., J., Kanbach, G., Reppin, C., and Chupp, E. L. 1984 Nature 312, 623-625. 